\title{
ANÁLISIS DEL DESEMPEÑO DE CANDIDATOS A TRADUCTOR E INTÉRPRETE OFICIAL EN COLOMBIA ${ }^{1}$
}

\author{
Juan Felipe Zuluaga Molina \\ Gabriel Quiroz Herrera \\ Universidad de Antioquia \\ Medelín, Antioquia, Colombia
}

\begin{abstract}
Resumen: El ejercicio profesional de la traducción e interpretación oficial en Colombia está representado por el conglomerado de traductores e intérpretes que han superado las pruebas de traducción e interpretación que, según la legislación actual, dos universidades nacionales ofrecen al público en general. No obstante, existe poco reconocimiento social (Quiroz et al, 2015) y legal de ese conglomerado y del grupo de potenciales traductores oficiales que presentan las pruebas en el país. En el presente artículo, se describe, por medio de análisis cuantitativos, los resultados del examen de traducción e interpretación oficial de 134 candidatos inscritos desde 2011 hasta 2016 en el proceso de certificación de la Escuela de Idiomas de la Universidad de Antioquia. Los datos generales nos indican que al menos 1 de cada 10 candidatos al examen aprueba el examen, que las mujeres tienen un mejor desempeño en la prueba y que, en términos generales, el desempeño de los candidatos para el par de lenguas francés-español es superior al de los del par inglés-español. En cuanto a los errores, el tipo de error más común en todos los exámenes es el error terminológico. Desde el punto de vista de los tipos de pruebas y competencias, la interpretación, tanto a vista como alterna, se presenta como la competencia más compleja de superar para la mayoría de los candidatos.
\end{abstract}

Palabras clave: Traducción e interpretación oficial; Evaluación en traducción; Traducción en Colombia; Estatus del traductor en Colombia

\footnotetext{
${ }^{1}$ El presente proyecto de investigación está registrado ante el CODI según Acta del 52 de marzo 2017. Además, hace parte del proceso de investigación de la tesis de Maestría en Traducción de Juan Felipe Zuluaga Molina en la Universidad de Antioquia, Colombia, cuyo asesor es Gabriel Quiroz Herrera.
} 


\title{
SWORN TRANSLATORS AND INTERPRETERS CANDIDATES' PERFORMANCE ANALYSIS IN COLOMBIA
}

\begin{abstract}
Sworn Translation and Interpreting as a professional activity in Colombia is carried out by those translators and interpreters that have approved the translation and interpreting examinations offered by two universities according to the law to the public at large. However, there is few social and legal visibility (Quiroz et al, 2015) for these professional community and the group of potential sworn translators and interpreters.. In this article, the results of the application of a sworn translation and interpreting exam will be described by using a quantitative approach. 134 candidates from 2011 to 2016 were studied at the School of Languages examination in Universidad de Antioquia. The overview of results indicates that at least 1 out of 10 candidates approved the test; women tend to have a better outcome, generally speaking; candidates working in the language pair French-Spanish perform better than those from the language pair English-Spanish. In terms of error analysis, the most common error in both sections is the terminological error. Besides, from the point of view of types of tests and competences, it is important to mention that interpreting is the most complex competence to overcome to most candidates.
\end{abstract}

Key words: Sworn translation and interpreting; Translation testing; Translation in Colombia; Translation status in Colombia.

\section{Introducción}

El oficio de la traducción e interpretación oficial, adoptado legalmente desde 1951 en Colombia según los decretos 382 y 2257 de 1951, es ejercido profesionalmente por quienes han superado las pruebas de idoneidad sobre el desempeño tanto en traducción como en interpretación. La evaluación de ambas competencias es, de hecho, la única vía de acceso a este oficio que existe en Colombia, tal como se menciona en los decretos anteriormente expuestos. El decreto 722 de 1982 es la norma en la cual se establecen los aspectos que se deben tener en cuenta a la hora de evaluar a los candidatos. 
No obstante, dicho decreto, que no refleja ya ni la práctica profesional, ni las necesidades del mercado, ni los avances teóricos en evaluación de traducciones, no ha tenido, desde hace mucho tiempo ya, un cambio significativo que le permita tener una vigencia como para ser un documento de referencia para los académicos y organizadores de dicho proceso de examinación.

Con miras a comprender de mejor manera la realidad de los procesos de certificación en traducción oficial en el país, es importante saber que el Ministerio de Relaciones Exteriores avala solamente a dos universidades en Colombia para el desarrollo de las pruebas, a saber, la Universidad de Antioquia de Medellín y la Universidad Nacional de Bogotá. Sin embargo, aun cuando es este Ministerio el que promulgó dichos avales, no es él ni ningún otro Ministerio quien regula el desempeño ni la certificación de los traductores ni trabaja en conjunto con las comisiones para comprender y mejorar los procesos de evaluación a potenciales traductores e intérpretes oficiales ni el ejercicio mismo de este oficio.

El panorama antes descrito permite entonces avanzar en la hipótesis de la necesidad latente de construir comunidad y reflexión académica acerca de los procesos de evaluación en traducción oficial o, al menos, contribuir al descubrimiento y reconocimiento de los procedimientos actuales de evaluación y calificación de traductores e intérpretes que se utilizan en Colombia, para posteriormente pensar en su pertinencia y aplicabilidad o replicabilidad en el territorio o en el conjunto latinoamericano.

En el presente artículo, se describe la estrategia de evaluación del Examen para Traductor e Intérprete Oficial (ETIO) administrado por la Escuela de Idiomas de la Universidad de Antioquia, Colombia. Así mismo, busca discutir la forma cómo se ha utilizado y los resultados que ha arrojado su implementación en un grupo de 134 aspirantes a Traductores e Intérpretes Oficiales durante las diferentes convocatorias desde el 2011 hasta el 2016. Las pretensiones o intenciones generales del artículo se basan en la acuciante necesidad de mejorar las prácticas de reconocimiento de las dificultades de la traducción en Colombia, por lo que el artículo busca, 
además de describir la estrategia de evaluación, descubrir el desempeño de los potenciales traductores oficiales en el país teniendo en cuenta datos demográficos básicos como sexo, edad, cohorte del examen, educación, ciudad, etc., y proponer soluciones o líneas de análisis posteriores a estas realidades desde perspectivas gremiales, académicas, contextuales y profesionales.

\section{Marco conceptual}

La evaluación en traducción, en los diferentes espacios en los que se aplica, ha sido, por mucho tiempo, un tema de estudio para varios teóricos dedicados al tema. Una buena cantidad de estrategias y de propuestas con respecto a la forma cómo debe evaluarse han surgido en diferentes espacios, como en el académico y el laboral, tanto como proceso como producto (Sager, Gouadec, Paramètres, Gouadec, Le Traducteur, Bachman, Deslile, Pym, Hurtado, Traducción, Kussmaul, Nord, Chamosa, House, Larose, Waddington, Reiss, Martínez \& Hurtado, Williams, Translation Quality Assessment; Williams, Translation Quality Assessment: An Argumentation, entre otros autores que exploran el tema desde diferentes perspectivas teóricas y metodológicas). Es importante poner de relieve que nos centraremos en indicar algunos aspectos generales de la experiencia de aplicación o utilización de un instrumento de evaluación basado en el producto. Para fines de comprensión conceptual, aceptamos comúnmente la definición ampliada de error dada por Hurtado Albir: "equivalencia inadecuada para la tarea traductora encomendada" (280, Traducción), una definición que se ajusta bien a las pretensiones del presente artículo, puesto que nuestro trabajo se centrará en el momento posterior a la utilización de la estrategia de evaluación, es decir a los resultados arrojados luego de la presentación del examen, y no a una discusión de los aspectos teóricos o metodológicos ligados a la construcción de la estrategia. 


\subsection{Algunos antecedentes del examen para traductor e intérprete oficial en Colombia}

La figura de "traductor e intérprete oficial” está presente legalmente en Colombia desde 1951 cuando desde el Estado colombiano se crea mediante los decretos 382 y 2257 . Para esta época la ley define que la función principal de un traductor e intérprete oficial ${ }^{2}$ sería:

Traducir de cualquier idioma al castellano o viceversa, todos los documentos cuya traducción y autenticación sean solicitadas por el público para que presten mérito oficial ante las autoridades, y servir de intérpretes orales en los casos señalados por la ley. (DECRETO 382, 2012, s/p) (DECRETO 2257, 1951,s/p)

Por la misma línea, es a través de la legislación que se determina que este potencial traductor e intérprete oficial deberá certificarse a través de un examen, el cual, tal como lo dice Stejskal:

[...] was first handled by a special institute that was simultaneously responsible for preparing candidates for qualification (IEI). After a series of irregularities were reported, certification was passed, in 1988, to the State Universidad Nacional Modern Languages Department in an attempt to fight corruption and to add a measure of academic judgment. (2013, p. 8)

Retomando así la historia y construcción indicada anteriormente, es apenas en 1988 que la Universidad Nacional inicia labores de certificación y, mucho después, se le une la Universidad de

${ }^{2}$ En Colombia, no se separan las dos profesiones en la ley (traductor - intérprete). Por lo tanto, la palabra "traductor" se refiere tanto al traductor como al intérprete oficial.

Cad. Trad., Florianópolis, v. 38, n $^{0}$ 2, p. 263-293, mai-ago, 2018 
Antioquia (2011), lo que muestra el panorama de tan solo dos instituciones avaladas por el Ministerio de Relaciones Exteriores para realizar este tipo de certificaciones y, especialmente, de un desarrollo administrativo aún muy joven (apenas dos décadas de trabajo continuo en ambas comisiones).

El decreto 722 de 1982, art. 2, define aspectos temáticos acerca de la composición del examen que deberían impartir las instituciones avaladas. En el decreto se establece que:

Los exámenes para los aspirantes a intérpretes oficiales versarán sobre dos evaluaciones, una oral y otra escrita. En la oral se tendrán presentes los siguientes aspectos: a) Habilidad del candidato para leer en la lengua extranjera con corrección en la pronunciación y en la entonación. b) Habilidad para traducción consecutiva de la lengua extranjera al español y viceversa, con exactitud en las ideas y fluidez en el campo del idioma. c) Habilidad para captar el sentido general y retener las ideas principales de un texto escuchado. En la evaluación escrita se tendrán en cuenta los siguientes aspectos: a) Habilidad del candidato para reconocer cognados falsos. b) Habilidad para traducir del idioma extranjero al español y viceversa con exactitud en el sentido, corrección ortográfica y naturalidad en el manejo de los dos idiomas. c) Habilidad en el uso del diccionario. (DECRETO 722,1982, s/p)

Desde entonces, tan solo la Ley Antitrámites 962 de 2005, Art. 33 reglamenta las nuevas disposiciones sobre el examen en cuanto a la apertura de instituciones que pueden proveer el examen y dicta que:

Toda persona que aspire a desempeñar el oficio de Traductor e Intérprete Oficial deberá aprobar los exámenes que sobre la materia dispongan las universidades públicas y privadas que cuenten con facultad de idiomas debidamente 
acreditadas y reconocidas por el ICFES o la entidad que tenga a cargo tal reconocimiento. (LEY 962, 2005, s/p)

Se trata de una ley que poco habla o contesta acerca de lo que se presenta en el citado decreto y que no específica o contribuye a la explicitación, de ninguna manera, de la actividad o el oficio de traducción oficial. De hecho, en la actualidad, este examen se aplica a las personas interesadas en presentar la prueba que los habilite para desempeñar la función de traductor e intérprete oficial (TIO) y ha sido configurada en ambas universidades, en muchas ocasiones, con interpretaciones propias y no colectivas, es decir sin consenso entre las Comisiones que lideran el proceso de administración del examen, de conformidad con la legislación colombiana, es decir, está dirigida a un público general y no a un público experto específico. Por ejemplo, una persona sin ningún tipo de estudios secundarios o universitarios podría presentarse al examen, al igual que un menor de edad, etc.

\section{La certificación de TIO en la Escuela de Idiomas de la Universidad de Antioquia}

Por un lado, tal como se ha indicado anteriormente, en Colombia hay dos instituciones avaladas para llevar a cabo el Examen para Traductor e Intérprete Oficial. En la Universidad Nacional de Bogotá, tal como aparece en su página, se comenta que:

Mediante el Decreto 1677 del 27 de junio de 1997, el Departamento Administrativo de la Función Pública fusiona el Instituto Electrónico de Idiomas a Universidad Nacional de Colombia y éste queda adscrito al Departamento de Lenguas Extranjeras. Desde este momento la aplicación de los Exámenes de Traducción e Interpretación Oficial queda a cargo del Departamento de Lenguas Extranjeras. Desde diciembre de 1998 la Universidad Nacional de Colombia 
ha venido aplicando los exámenes dos veces al año. ${ }^{3}$ (DECRETO 1677, 1997, s/p)

Así mismo, se comenta en su página web que dicho examen consta de dos pruebas escritas y dos pruebas orales (traducción interpretación) $)^{4}$.

Por otro lado, desde 2011, la creación oficial del Examen en la Universidad de Antioquia, se ha mantenido una estructura de examen en la cual se entiende que ser un Traductor e Intérprete Oficial implica, por definición, poseer una doble competencia: competencia oral (interpretación) y competencia escrita (traducción escrita) según se concluye o interpreta de las funciones expuestas en el decreto 722 de 1982. Esta comprensión es bastante similar a la que tiene la Comisión del Examen en Bogotá debido a que también evalúa ambas competencias en condiciones parecidas a las que serán expuestas a continuación, aunque con variaciones prácticas y evaluativas. Teniendo en cuenta entonces la lógica anteriormente expuesta, cada candidato debe presentar y aprobar dos secciones (traducción e interpretación) para superar la prueba, tal como se describe a continuación:

- La sección 1 (interpretación) consiste tanto en la interpretación a vista (ver la definición de Hurtado, 82, Traducción) como en la alterna (Hurtado, 82, Traducción) de un texto de carácter científico-técnico y de un texto de carácter institucional, lo cual debe tomar aproximadamente 10 minutos por prueba.

- La prueba 2 requiere la traducción de un texto científicotécnico y de un texto institucional, cada uno de 300 palabras aproximadamente, lo cual debe tomar una hora por texto como máximo.

${ }^{3} \mathrm{http} / / /$ www.extio.unal.edu.co/reg/antecedentes.php.

${ }^{4}$ http://www.extio.unal.edu.co/reg/examen.php. 
Con respecto a los requisitos de entrada o acceso al examen, es importante comentar que, debido a la inexistencia de estos dentro de la ley, no se exige la certificación de ninguna experiencia en traducción o interpretación, ni ningún título relacionado con las lenguas o el área. Esto contradice uno de los elementos recomendados durante el proceso de inscripción de los candidatos al examen (en un artículo que aparece en la Revista Babel, 2014; y otro que aparece en la Revista Núcleo, 2013 de los autores del presente artículo; se comentan especificidades de los diferentes procesos y perfiles de los candidatos al examen y se amplía más la discusión esbozada). Lo que interesa de este elemento dentro de la conversación es que el tener una carrera universitaria relacionada con la traducción o al menos con las lenguas o la lingüística, que son elementos que permiten tener un mejor desempeño en el examen (Quiroz et al, Candidatos), son requisitos no obligatorios para la presentación al examen, por lo que conviene pensar en la diversidad del público que presenta el examen no solo en formación profesional, sino también en edad, origen y ocupación (Quiroz et al, Candidatos).

\subsection{Acerca de la estrategia de evaluación implementada}

Con el fin de certificar el desempeño de los candidatos en las dos competencias anteriormente mencionadas (traducción e interpretación), el examen implementa una estrategia de evaluación sumativa en la cual se marcan, comentan y anotan los errores detectados en el aspirante y se organizan de acuerdo con su gravedad y tipificación en una hoja de cálculo.

La propuesta de evaluación surge en la práctica de la norma SAE J2450, un instrumento de evaluación que fue creado en 2005 para la industria automotriz con la intención de evaluar las traducciones que surgían en este ámbito y asegurar su calidad y pertinencia según las necesidades del cliente. La elección de este instrumento se basa principalmente en la constatación de que es una norma que, incluso estando inmersa en un escenario global y profesional, evidenciaba rasgos comunes entre los académicos de la 
evaluación en traducción e interpretación en lo que respecta sobre todo a la sistematicidad e importancia en el marcaje de errores y la necesidad de promover una garantía mínima en la calidad de este tipo de productos o servicios lingüísticos. En la norma se comenta que, en general:

The objective of the proposed quality metric is to establish a consistent standard against which the quality of translation of automotive service information can be objectively measured regardless of the source language, regardless or the target language, and regardless of how translation is performed (i.e., human translation or machine translation). The metric allows an evaluator to tag errors in a translation and compute a weighted, numeric score that represents the quality of the translation. (SAE J2450, 2005, s/p)

En el mismo documento se explica que la constitución de la norma es de:

- Seven error categories

- Two sub-categories

- Two meta-rules to help decide ambiguities on the assignment of an error to the categories and sub-categories

- Numeric weights (1)

En la imagen 1, se observa el instrumento terminado y utilizado por los expertos en traducción automotriz con el fin de controlar estos procesos: 
TABLE 1-ERROR CATEGORIES, CLASSIFICATIONS, AND WEIGHTS

\begin{tabular}{lcc}
\hline \multicolumn{1}{c}{$\begin{array}{c}\text { Category Name: } \\
\text { (abbreviation) }\end{array}$} & $\begin{array}{c}\text { Sub-Classification: } \\
\text { (abbreviation) }\end{array}$ & $\begin{array}{c}\text { Weight: } \\
\text { serious/minor }\end{array}$ \\
\hline a. Wrong Term (WT) & serious (s) & $5 / 2$ \\
b. Syntactic Error (SE) & minor (m) & $4 / 2$ \\
c. Omission (OM) & & $4 / 2$ \\
d. Word Structure or Agreement Error & & $4 / 2$ \\
(SA) & & $3 / 1$ \\
e. Misspelling (SP) & & $2 / 1$ \\
f. Punctuation Error (PE) & & $3 / 1$ \\
g. Miscellaneous Error (ME) & & \\
\hline
\end{tabular}

Imagen 1 - Categorías, clasificaciones y peso de los errores según la norma SAE J2450. Fonte: SAE J2450

\subsection{La plantilla de evaluación}

A continuación, se muestra la plantilla de evaluación utilizada para el proceso de revisión de los exámenes. Obsérvese que el instrumento en cuestión es una versión final de las reflexiones y definiciones de la Comisión para poder suplir las necesidades profesionales y evaluativas propias del contexto basadas en la norma anterior pero con la reinterpretación y posterior adhesión de categorías o elementos ausentes en la norma desde la cual se partió. Es importante decir que no es el foco principal del presente artículo generar una discusión acerca de la pertinencia del instrumento de evaluación aplicado para las pruebas sino que, por una línea posterior, se intenta observar cuáles han sido los descubrimientos que se han hecho por medio de la utilización de esta estrategia.

Para comprender mejor el contenido de la plantilla es importante entender que un candidato al examen tiene 100 puntos de partida para aprobar el examen. Estos puntos de partida se van quitando, en la medida en que el candidato va cometiendo errores dentro del examen. No obstante, la disminución de errores no es de uno en uno, es decir, no significa que si el candidato cometió tres errores en toda la prueba entonces su resultado final es 97 sino que, dependiendo del tipo de error y de su gravedad, se disminuyen puntos a ese conglomerado general por prueba y luego se hace un promedio 
de su desempeño negativo para así determinar los puntos que le serán restados a su promedio general sobre 100. Para dar un ejemplo práctico: un candidato aprueba el examen siempre y cuando su resultado final sea mayor a 87. Así, un candidato que cometa 5 errores terminológicos graves en cada una de las pruebas tendrá una reducción de 15 puntos por prueba (puesto que la valoración en puntos de un error terminológico grave es de 3 ) y en promedio general de 15 igualmente $(5 * 3 * 4 / 4) \mathrm{y}$, por ende, su resultado final es $85 / 100$ (100-15) que es el resultado de haber disminuido 15 puntos (que es el promedio general de desempeño negativo en la prueba) al total general posible de aprobación (100). El resultado final para un candidato con este desempeño sería no aprobado.

\begin{tabular}{|l|l|l|l|}
\hline \multicolumn{4}{|l|}{ Tabla de categorías de errores y su valoración en puntos negativos } \\
\hline Valoración en puntos negativos: & Error grave & Error leve & Abreviatura \\
\hline Tipología de errores & $\begin{array}{l}\text { Puntos } \\
\text { negativos }\end{array}$ & $\begin{array}{l}\text { Puntos } \\
\text { negativos }\end{array}$ & \\
\hline Omisión de párrafo & $\mathbf{1 1}$ & $\mathbf{3}$ & OP \\
\hline Error de léxico & $\mathbf{1}$ & $\mathbf{1}$ & EL \\
\hline Error sintáctico & $\mathbf{2}$ & $\mathbf{1}$ & ES \\
\hline Error terminológico & $\mathbf{3}$ & $\mathbf{1}$ & ET \\
\hline Error ortográfico & $\mathbf{3}$ & $\mathbf{1}$ & EO \\
\hline Error de puntuación & $\mathbf{2}$ & $\mathbf{1}$ & EP \\
\hline Error de secuencia & $\mathbf{4}$ & $\mathbf{1}$ & ES \\
\hline Error de registro & $\mathbf{2}$ & $\mathbf{1}$ & ER \\
\hline Error de transferencia & $\mathbf{3}$ & $\mathbf{1}$ & ETR \\
\hline Errores misceláneos & $\mathbf{2}$ & $\mathbf{1}$ & EM \\
\hline $\begin{array}{l}\text { Errores propios de interpretación: } \\
\text { Entonación }\end{array}$ & $\mathbf{3}$ & $\mathbf{1}$ & INT \\
Pronunciación & $\mathbf{I N T E}$ \\
Acento & Ritmo & $\mathbf{3}$ & $\begin{array}{l}\text { INTP } \\
\text { INTA } \\
\text { INTR }\end{array}$ \\
\hline
\end{tabular}

Tabla 1 - Tabla de categorías de errores y su valoración en puntos negativos. 


\subsubsection{Tipos de errores}

A continuación, se explica cada uno de los errores y se ejemplifica cada caso. Para estas aclaraciones se explicarán algunas cuestiones técnicas relacionadas con la pertinencia de esta tipología. Los ejemplos han sido tomados de los exámenes presentados en las diferentes convocatorias realizadas, aunque sólo algunos errores han sido simulados con el objetivo de materializar en la práctica la forma cómo se comete un error en los textos que son utilizados para el examen. Los datos o informaciones de definición de la tipología se extrajeron de la "Guía para el Examen para Traductor e Intérprete Oficial" de la Universidad de Antioquia, creada por un grupo de expertos en traducción tanto a nivel académico como profesional, luego de hacer un abordaje teórico y metodológico del tema y basados en sus experiencias con respecto a traducción oficial. Es importante evidenciar que, el juicio por la levedad o gravedad de un error es potestad del jurado debido a que el criterio dado por la Comisión para determinarlo se basa simplemente en la afección del mismo en el sentido del texto. Es decir que, cuando un error afecta significativamente el sentido que se quería comunicar en el texto original hay lugar a un error grave y cuando simplemente es un error que no afecta el sentido general de lo que se quería comunicar se considera un error leve.

3.2.1.1 OMISIÓN: Se entiende por omisión, el hecho que un aspirante haya dejado de traducir o reproducir algún segmento del texto, trátese de una frase, oración, párrafo, tabla, diagrama, foto, formato o dibujo que induzca a algún tipo de incomprensión del texto o a la ausencia de elementos presentes dentro del original. De esta manera se pasará a identificar si trata de un error grave, cuando se trata de la omisión de un párrafo de 4 líneas o más, por ejemplo; y de un error leve cuando se trata de una frase, por ejemplo. Vázquez-Ayora (358) habla acerca de la omisión, sus formas y especificidades. 


\begin{tabular}{|l|l|}
\hline $\begin{array}{l}\text { Lengua original: } \\
\text { Inglés }\end{array}$ & $\begin{array}{l}\text { Built-in ADSL modem provides direct, always-on } \\
\text { Internet connectivity and multi-user access sharing } \\
\text { at speeds up to } \mathbf{1 4 0} \text { times faster than dial-up }\end{array}$ \\
\hline $\begin{array}{l}\text { Lengua meta: } \\
\text { Español }\end{array}$ & $\begin{array}{l}\text { El modem integrado ADSL provee conectividad } \\
\text { continua y directa a Internet y acceso multiusuario } \\
\text { compartido a velocidades mayores que las de la } \\
\text { línea conmutada. }\end{array}$ \\
\hline Error & Omisión: error grave por omitir el número. \\
\hline Corrección & $\begin{array}{l}\text { El modem integrado ADSL provee conectividad } \\
\text { continua y directa a Internet y acceso multiusuario } \\
\text { compartido a velocidades mayores a } \mathbf{1 4 0} \text { veces las } \\
\text { de la línea conmutada. }\end{array}$ \\
\hline
\end{tabular}

Tabla 2 - Ejemplo de error: omisión

3.2.1.2 ERROR DE LÉXICO: En esta categoría se incluyen errores de vocabulario básicos que no entren en las categorías de error terminológico.

\begin{tabular}{|l|l|}
\hline $\begin{array}{l}\text { Lengua original: } \\
\text { Español }\end{array}$ & $\begin{array}{l}\text { En este último cargo le correspondió liderar la aplicación } \\
\text { de la Política de Seguridad Democrática. }\end{array}$ \\
\hline $\begin{array}{l}\text { Lengua meta: } \\
\text { Inglés }\end{array}$ & $\begin{array}{l}\text { In this last post, he was in charge of leading the } \\
\text { Democratic Security Policy's application. }\end{array}$ \\
\hline Error & Error léxico: "post" tomado como "position" \\
\hline Corrección & $\begin{array}{l}\text { In this last position, he was in charge of leading the } \\
\text { Democratic Security Policy's application. }\end{array}$ \\
\hline
\end{tabular}

Tabla 3 - Ejemplo de error de léxico.

3.2.1.3 ERROR SINTÁCTICO: El error sintáctico se comprende de tres maneras, (1) en la presencia de una estructura sintáctica incorrecta; (2) cuando hay falta de coherencia en la redacción en casos específicos de género, número y tiempo; y (3) cuando se observa falta de cohesión semántica en un párrafo, en una oración $o$ en una frase. 


\begin{tabular}{|l|l|}
\hline $\begin{array}{l}\text { Lengua original: } \\
\text { español }\end{array}$ & El 20 de junio de 2010 \\
\hline Lengua meta: inglés & In June 20th, 2010 \\
\hline Error & $\begin{array}{l}\text { Error sintáctico grave: } \text { in }=\text { on. Presencia de una } \\
\text { estructura sintáctica incorrecta de la lengua meta. }\end{array}$ \\
\hline Corrección & On June 20th, 2010. \\
\hline
\end{tabular}

Tabla 4 - Ejemplo de error sintáctico.

3.2.1.4 ERROR TERMINOLÓGICO: El error terminológico tiene que ver con la utilización de un término incorrecto o no usual. Así mismo se entiende por este tipo de error, el manejo equivocado de un acrónimo, una sigla, una abreviatura, un nombre propio o una unidad de medida. Con respecto a las incorrecciones terminológicas ya Newmark $(145,148,198)$ había adelantado una importante sustentación al respecto.

\begin{tabular}{|l|l|}
\hline $\begin{array}{l}\text { Lengua original: } \\
\text { español }\end{array}$ & La ONG contribuyo en el rescate. \\
\hline Lengua meta: inglés & The ONG helped in the rescue. \\
\hline Error & $\begin{array}{l}\text { Error terminológico: en inglés la traducción de ONG } \\
\text { es NGO. }\end{array}$ \\
\hline Corrección & The NGO helped in the rescue. \\
\hline
\end{tabular}

Tabla 5 - Ejemplo de error terminológico.

3.2.1.5 ERROR ORTOGRÁFICO: En el error ortográfico se tienen en cuenta las dificultades de escritura que hayan sido causales de incomprensión del texto o no. En este sentido, una grafía incorrecta o una mala acentuación podría entenderse como un error de este tipo.

\begin{tabular}{|l|l|}
\hline Lengua original: inglés & The government gave three cars to them. \\
\hline Lengua meta: español & El gobierno les dió tres carros a ellos. \\
\hline
\end{tabular}




\begin{tabular}{|l|l|}
\hline Error & $\begin{array}{l}\text { Error ortográfico: la palabra "dio" no lleva tilde } \\
\text { en la "o" }\end{array}$ \\
\hline Corrección & El gobierno les dio tres carros a ellos. \\
\hline
\end{tabular}

Tabla 6 - Ejemplo de error ortográfico.

3.2.1.6 ERROR DE PUNTUACIÓN: en el error de puntuación se sancionan todos aquellos momentos en los que el aspirante deje de poner cualquier signo de puntuación correspondiente a la traducción que esté realizando.

\begin{tabular}{|l|l|}
\hline Lengua original: inglés & Did the FDA approve that? \\
\hline Lengua meta: español & Aprobó la FDA esto? \\
\hline Error & $\begin{array}{l}\text { Error de puntuación: en español se empieza y se } \\
\text { cierra la pregunta con los signos de interrogación }\end{array}$ \\
\hline Corrección & ¿Aprobó la FDA esto? \\
\hline
\end{tabular}

Tabla 7 - Ejemplo de error de puntuación.

3.2.1.7 ERROR DE SECUENCIA: para este criterio se ha decidido tener en cuenta todas las incoherencias o falta de designación o sistematicidad estilística que presente el aspirante en el examen que tengan que ver con eventos, procesos o acciones.

\begin{tabular}{|l|l|}
\hline $\begin{array}{l}\text { Lengua original: } \\
\text { inglés }\end{array}$ & $\begin{array}{l}\text { Keep batteries out of the reach of children. } \\
\text { Remove batteries if storing over 30 days. }\end{array}$ \\
\hline Lengua meta: español & $\begin{array}{l}\text { Mantenga la batería fuera del alcance de los niños. } \\
\text { Retirar las baterías si han estado almacenadas por } \\
\text { más de 30 días. }\end{array}$ \\
\hline Error & $\begin{array}{l}\text { Error de secuencia: no hay secuencia o sistematicidad } \\
\text { en la utilización del verbo del principio de la frase. } \\
\text { Mantenga/Retirar. }\end{array}$ \\
\hline Corrección & $\begin{array}{l}\text { Mantenga la batería fuera del alcance de los niños. } \\
\text { Retire las baterías si han estado almacenadas por más } \\
\text { de 30 días. }\end{array}$ \\
\hline
\end{tabular}

Tabla 8 - Ejemplo de error de secuencia.

Cad. Trad., Florianópolis, v. 38, $\mathrm{n}^{0}$ 2, p. 263-293, mai-ago, 2018 
3.2.1.8 ERROR DE REGISTRO: este tipo de error se identifica cuando se observa en el texto una mala utilización de la lengua en términos culturales o de variedades dialectales.

\begin{tabular}{|l|l|}
\hline $\begin{array}{l}\text { Lengua original: } \\
\text { inglés }\end{array}$ & $\begin{array}{l}\text { President Santos said: "I think President Obama and } \\
\text { Bush have been my friends..." }\end{array}$ \\
\hline Lengua meta: español & $\begin{array}{l}\text { El Presidente Santos dijo: "Pienso que el Presidente } \\
\text { Obama y Bush han sido mis camaradas..." }\end{array}$ \\
\hline Error & $\begin{array}{l}\text { Error registro: la palabra "camarada" no es } \\
\text { pertinente para la traducción de la forma "friend" } \\
\text { debido a la evidente inadecuación dentro del } \\
\text { contexto político o presidencial. }\end{array}$ \\
\hline Corrección & $\begin{array}{l}\text { El Presidente Santos dijo: "Pienso que el Presidente } \\
\text { Obama y Bush han sido mis amigos..." }\end{array}$ \\
\hline
\end{tabular}

Tabla 9 - Ejemplo de error de registro.

3.2.1.9 ERROR DE TRANSFERENCIA: se trata de momentos en los que el aspirante deja de traducir una parte del texto original y/o lo deja tal cual, lo que podría considerarse un error de transferencia.

\begin{tabular}{|l|l|}
\hline Lengua original: inglés & $\begin{array}{l}\text { True Firewall using Stateful Packet Inspection (SPI) } \\
\text { and Intrusion Control features Denial of Service } \\
\text { protection from hacker attacks }\end{array}$ \\
\hline Lengua meta: español & $\begin{array}{l}\text { El True Firewall cuenta con Stateful Packet } \\
\text { Inspection (SPI) e Intrusion Control features } \\
\text { Denial of Service contra ataques de hackers. }\end{array}$ \\
\hline Error & $\begin{array}{l}\text { Error de transferencia: error grave de no traducción } \\
\text { que sí tiene equivalente al español. }\end{array}$ \\
\hline
\end{tabular}

Tabla 10 - Ejemplo de error de transferencia.

3.2.1.10 ERRORES MISCELÁNEOS: los errores misceláneos son comprendidos en tres categorías: la primera (1) se define como traducción literal, de una frase o de una oración, lo que puede inducir a la incomprensión del texto o un cambio de sentido 
evidente; la segunda (2) es la sobretraducción, error en el cual se agregan elementos a la traducción que no eran necesarios y que tendrían que ser omitidos aunque podrían ser causantes de incomprensión para el aspirante; y la tercera (3) la subtraducción, que hace referencia a la acción contraria del ítem anterior.

\begin{tabular}{|l|l|}
\hline Lengua original: inglés & $\begin{array}{l}\text { Ease of Use Advantages } \\
\text { User interface matches your local language } \\
\text { (English, French, German or Italian)... }\end{array}$ \\
\hline Lengua meta: español & $\begin{array}{l}\text { Ventajas de uso fácil } \\
\text { Interfaz de usuario en su idioma (inglés, francés, } \\
\text { alemán, italiano o español)... }\end{array}$ \\
\hline Error & Error misceláneo: sobretraducción innecesaria. \\
\hline Corrección & $\begin{array}{l}\text { Ventajas de uso fácil } \\
\text { Interfaz de usuario en su idioma (inglés, francés, } \\
\text { alemán, o italiano)... }\end{array}$ \\
\hline
\end{tabular}

Tabla 11 - Ejemplo de error misceláneo.

3.2.1.11 ERRORES DE INTERPRETACIÓN: para la caracterización de los errores de interpretación se tienen en cuenta cuatro aspectos: entonación, acento, ritmo y pronunciación. Estos aspectos serán tenidos en cuenta siempre y cuando tengan una incidencia significativa en el sentido debido a que es difícil su comprensión o a que comunican una idea diferente a la que se desea comunicar.

\begin{tabular}{|l|l|}
\hline Lengua original: español & Ganadores \\
\hline Lengua meta: inglés & Winners \\
\hline Error en la pronunciación & /Wainers/ \\
\hline Descripción & $\begin{array}{l}\text { Error de interpretación: mala pronunciación } \\
\text { que impide la comprensión de la palabra en el } \\
\text { contexto específico. }\end{array}$ \\
\hline
\end{tabular}

Tabla 12 - Ejemplo de error de interpretación 


\section{Análisis cuantitativo del desempeño de los candidatos en el ETIO}

Luego de una descripción detallada de la composición y estructura del examen, de la Universidad de Antioquia, en la presente sección y hacia adelante mostraremos los resultados de los candidatos que han presentado el examen en las diferentes convocatorias. En el siguiente análisis se ofrece un breve panorama del desempeño general según diferentes criterios de análisis y luego una especificación acerca de los errores más comunes que cometen los aspirantes. El objetivo de la presente sección es contribuir a la comprensión de los perfiles de los potenciales traductores e intérpretes oficiales, y descubrir algunos rasgos comunes de los mismos, así como describir en la práctica el comportamiento de los errores en este tipo de exámenes con la utilización de la estrategia antes descrita.

\subsection{Datos generales}

Desde 2011 hasta 2016, se han presentado 134 candidatos, de los cuales 14 han aprobado el examen, es decir, apenas el 10\% aproximadamente de los participantes. Se observa también que, luego del análisis y las observaciones de los datos de la Comisión del Examen, la distribución por sexo dentro del examen es más o menos pareja (72 hombres y 62 mujeres se han presentado al examen) y que, por el contrario, la tendencia de aprobación está más del lado femenino en términos relativos (de las 14 personas que han aprobado el examen, 7 son hombres y 7 son mujeres). Es también interesante comentar datos como el hecho de que se han presentado sólo 18 candidatos a las pruebas de francés y 116 a las de inglés, algo que reproduce lo comentado en anteriores análisis (Quiroz et al, Panorama) acerca de la presencia inminente del inglés en el territorio y su permanencia como una lengua de actividad frecuente en el área de la traducción e interpretación en Colombia. Así mismo, es importante comentar que, de los 14 nuevos traductores certifica- 
dos, apenas 4 de ellos lo son en el par de lenguas inglés-español y 10 de ellos en el par francés-español. Esto nos indica una marcada tendencia positiva en lo que respecta al desempeño de los candidatos en las pruebas de francés y, especialmente, en el segmento de los hombres aunque no de forma relativa (de los 10 nuevos traductores e intérpretes oficiales en francés, 5 son hombres y 4 mujeres).

Así como los datos anteriormente ilustrados, muchos otros confirmarán la idea o la ejemplificación del perfil y el desempeño de los candidatos en las pruebas de calificación desde otras perspectivas. Simplemente, queríamos iniciar esta sección dando algunas líneas generales de apoyo a la discusión que ya ha sido adelantada en las diferentes publicaciones referidas (Quiroz et al, Estatus).

\subsection{Análisis general de desempeño por convocatoria}

La Tabla 13 evidencia en términos generales el desempeño de los candidatos en las convocatorias en las dos competencias evaluadas. Como datos interesantes, hay que comentar el hecho de que las pruebas de interpretación, por su complejidad y "temor" comentado por los mismos candidatos, se presenten como las pruebas en las que los candidatos tienen un desempeño negativo. Obsérvese que en un ponderado definido sobre 100, el desempeño en las pruebas de traducción es mayor al desempeño porcentual general en ambas competencias. La Tabla 13 también nos permite ahondar en la discusión de los perfiles y el desempeño de los candidatos, sobre todo cuando observamos que hay muchas convocatorias en las que el desempeño general está por debajo de 70, esto teniendo en cuenta que el umbral de aprobación está definido en un mínimo de 87. Es más, observando detenidamente lo que se presenta en la Tabla, apenas en tres de las diez convocatorias el desempeño general ha sido mayor a 70/100. Otro dato interesante que aporta insumos a la comprensión del comportamiento de este proceso de certificación es el hecho de que, de forma relativa, el desempeño de los aspirantes es mejor en las convocatorias que se realizan en el primer semestre del año... ¿Por qué? 


\begin{tabular}{|c|c|c|c|c|c|c|}
\hline \# & 葛 & 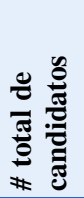 & $\begin{array}{l}\text { Desempeño } \\
\text { porcentual en } \\
\text { interpretación } \\
\text { (sobre 100) }\end{array}$ & $\begin{array}{l}\text { Desempeño } \\
\text { porcentual } \\
\text { en } \\
\text { traducción } \\
\text { (sobre 100) }\end{array}$ & $\begin{array}{l}\text { Desempeño } \\
\text { porcentual } \\
\text { general } \\
\text { (sobre 100) }\end{array}$ & $\begin{array}{l}\text { \# de } \\
\text { aprobados }\end{array}$ \\
\hline 1 & $2011 / 1$ & 14 & 47,3 & 64,1 & 55,7 & 1 \\
\hline 2 & $2011 / 2$ & 12 & 66,3 & 73,6 & 70 & 0 \\
\hline 3 & 2012 & 9 & 55,4 & 63 & 59 & 1 \\
\hline 4 & $2013 / 1$ & 15 & 92 & 68 & 81 & 3 \\
\hline 5 & $2013 / 2$ & 16 & 40,7 & 67,5 & 56 & 0 \\
\hline 6 & $2014 / 1$ & 17 & 55,8 & 62,1 & 59 & 1 \\
\hline 7 & $2014 / 2$ & 15 & 44,8 & 56,4 & 50,1 & 2 \\
\hline 8 & $2015 / 1$ & 15 & 62,5 & 72,6 & 67,5 & 2 \\
\hline 9 & $2015 / 2$ & 12 & 60,9 & 73,4 & 67 & 1 \\
\hline 10 & $2016 / 1$ & 8 & 70,2 & 79,1 & 77 & 3 \\
\hline Promedios & & & 59,59 & 67,98 & 64,23 & 1,4 \\
\hline
\end{tabular}

Tabla 13 - Análisis general del desempeño por competencia y por convocatoria.

La Tabla 14 permite identificar generalidades acerca de los perfiles de aprobación de los candidatos. Como datos interesantes de esta tabla, se observa por ejemplo que, aunque el desempeño general acumulado es mejor en el segmento de las mujeres $(64,5$ contra 62 de los hombres), los candidatos masculinos que aprueban el examen lo hacen con un mejor ponderado global que las mujeres (92,4 contra 90,4 de las mujeres). Es importante decir que, probablemente, unas pequeñas cifras decimales no son significativas para el lector pero, cuando se tiene en cuenta que son 62 mujeres y 72 hombres y que se está trabajando con datos obtenidos de la revisión en 11 categorías de 4 pruebas, es decir más de 40 variables, los datos pequeños o diferencias decimales empiezan a hablar por sí solas en lo que tiene que ver con el desempeño de los candidatos. 


\begin{tabular}{|l|l|}
\hline Criterio de desempeño & $\begin{array}{l}\text { Desempeño general en datos porcentuales sobre } \\
\text { 100 }\end{array}$ \\
\hline Candidatos de francés & 84,8 \\
\hline Candidatos de inglés & 60 \\
\hline Mujeres & 64,5 \\
\hline Hombres & 62 \\
\hline $\begin{array}{l}\text { Mujeres que aprobaron } \\
\text { el examen }\end{array}$ & 90,4 \\
\hline $\begin{array}{l}\text { Hombres que aprobaron } \\
\text { el examen }\end{array}$ & 92,4 \\
\hline
\end{tabular}

Tabla 14 - Datos generales de desempeño según algunos criterios etnográficos.

La Tabla 14 permite también avanzar aún más en la discusión acerca de la feminización de la profesión ya abordada por diferentes autores como Cachin (2007), Pym (1991), quien comenta que la profesión es en su mayoría femenina y que las discusiones de género al interior de la traducción son de corte históricamente femenino... Esto es interesante, puesto que para el segmento de traductores oficiales, al menos en el conglomerado de los 134 candidatos analizados, la tendencia es a que efectivamente las mujeres tengan un mejor desempeño en términos relativos dentro de la prueba y a que, a pesar de que no son la mayoría según el número de candidatos, tengan un mejor desempeño y posibilidad de aprobación en comparación con los hombres.

\subsection{Análisis general de desempeño en cada una de las secciones del examen}

Cuando nos detenemos en lo que ocurre con el desempeño por sección, encontramos que en la que más dificultad hay en el examen es en la de "interpretación alterna” (56/100). En esta una sección, 
el candidato escucha por unos segundos lo que otra persona dice en lengua materna o extranjera y luego se graba interpretando lo escuchado. Esta realidad no difiere mucho de lo que se observa en la cotidianidad en traducción en Colombia, donde encontramos cifras altas en lo que respecta al cobro de servicios de este tipo.¿Qué ocurre entonces con la preparación o el desempeño en este tipo de pruebas? ¿Habría que repensar esa inseparable condición de doble competencia en traducción e interpretación para el segmento de traductores e intérpretes oficiales?

\begin{tabular}{|l|l|l|l|l|l|}
\hline$\#$ & Convocatoria & $\begin{array}{l}\text { Interpretación } \\
\text { a vista }\end{array}$ & $\begin{array}{l}\text { Interpretación } \\
\text { alterna }\end{array}$ & $\begin{array}{l}\text { Traducción } \\
\text { científico- } \\
\text { técnica }\end{array}$ & $\begin{array}{l}\text { Traducción } \\
\text { institucional }\end{array}$ \\
\hline 1 & $2011 / 1$ & 47,4 & 47,2 & 76,2 & 51,9 \\
\hline 2 & $2011 / 2$ & 70,2 & 62,2 & 79,7 & 67,5 \\
\hline 3 & 2012 & 53,5 & 57,3 & 69,6 & 56,27 \\
\hline 4 & $2013 / 1$ & 93,76 & 90,8 & 66,06 & 71,03 \\
\hline 5 & $2013 / 2$ & 46,18 & 35,1 & 67,0 & 68,03 \\
\hline 6 & $2014 / 1$ & 68,9 & 42,7 & 68,7 & 55,4 \\
\hline 7 & $2014 / 2$ & 51,4 & 37,7 & 61,5 & 51,3 \\
\hline 8 & $2015 / 1$ & 62,67 & 62,3 & 75,8 & 69,4 \\
\hline 9 & $2015 / 2$ & 60,8 & 60,9 & 71,3 & 75,4 \\
\hline 10 & $2016 / 1$ & 71,45 & 69,04 & 80,6 & 77,2 \\
\hline Promedios & & 62,626 & 56,524 & 71,646 & 64,343 \\
\hline
\end{tabular}

Tabla 15 - Desempeño por sección del examen y convocatoria.

Otro aspecto que contribuye con la discusión es el desempeño en las pruebas de traducción científico-técnica. Este es un dato que constituye un hallazgo que contrasta con la sección que continúa en la que se observa que los errores más comunes en certificación de traducción oficial son los terminológicos. ¿A qué se debe que haya un buen desempeño en las pruebas de traducción científico-técnica, un segmento de la prueba en el que la cantidad de términos es grandísima, en vista del alto número de errores de terminología que hay en general en la prueba? Este y otros cuestionamientos siguen construyéndose a medida que presentamos los datos de desempeño de los 
candidatos y que avanzamos en la discusión del estado de la cuestión en el perfil de los candidatos a traductor e intérprete oficial.

\subsection{Análisis general de los errores más comunes (leves o graves)}

En la tabla número 15, se muestra el conglomerado de los errores de los candidatos que se han presentado a las diez convocatorias de la prueba en inglés y en francés. En esta tabla se logra observar que los errores más comunes son los errores terminológicos $(55,37 \%)$ seguidos de los errores sintácticos $(18,5 \%)$ y de los de omisión (6\%). La indicación inicial que nos dan estas estadísticas es un tema que bien serviría para llevar a cabo discusiones acerca de la forma correcta de preparación de candidatos al examen y de puntos de atención del examen para los candidatos lectores del presente artículo.

\begin{tabular}{|l|l|l|}
\hline Puesto & Tipo de error & $\begin{array}{l}\text { Porcentaje de desempeño } \\
\text { negativo }\end{array}$ \\
\hline $\mathbf{1}$ & Error terminológico & $55,37 \%$ \\
\hline $\mathbf{2}$ & Error sintáctico & $18,30 \%$ \\
\hline $\mathbf{3}$ & Omisión & $6,15 \%$ \\
\hline $\mathbf{4}$ & Errores misceláneos & $5,61 \%$ \\
\hline $\mathbf{5}$ & Error de transferencia & $4,89 \%$ \\
\hline $\mathbf{6}$ & Error de léxico & $4,19 \%$ \\
\hline $\mathbf{7}$ & Errores propios de interpretación & $1,54 \%$ \\
\hline $\mathbf{8}$ & Error de registro & $1,32 \%$ \\
\hline $\mathbf{9}$ & Error ortográfico & $1,07 \%$ \\
\hline $\mathbf{1 0}$ & Error de secuencia & $1,03 \%$ \\
\hline $\mathbf{1 1}$ & Error de puntuación & $0,49 \%$ \\
\hline
\end{tabular}

Tabla 16 - Tabla de jerarquía de los errores más comunes en el examen. 


\section{Cierre y recomendaciones finales de los hallazgos del presente estudio}

El estudio y análisis cuantitativo de los resultados y del desempeño de 134 aspirantes a traductores e intérpretes oficiales que han presentado el examen de certificación en diez convocatorias nos permite avanzar en la discusión acerca del perfil y el comportamiento de la traducción en Colombia.

En primer lugar, desde una perspectiva pragmática, el presente estudio da líneas claras de identificación o de descripción de las dificultades más evidentes que los traductores e intérpretes, al menos en el segmento de la traducción oficial, tienen. Estos hallazgos soportados con una cantidad alta de datos y el respaldo de la implementación de una estrategia de evaluación en traducción, podrían eventualmente influir en campos como el testing o la didáctica misma de la traducción, en tanto permiten afinar las técnicas de enseñanza y formación de traductores, y las técnicas de evaluación, entre otras. Por la misma línea, el artículo permite a los potenciales candidatos a certificarse identificar las dificultades de los aspirantes anteriores y buscar salidas o soluciones alternativas a estas dificultades. En tal sentido, algunas de las recomendaciones generales para los públicos objetivos mencionados anteriormente son: a. hay que replantearlas pruebas de interpretación pues se presenta como la competencia en la que hay un desempeño sobre todo negativo en un segmento ampliado de los candidatos analizados; b. hay que buscar alternativas complementarias de estudio para la preparación en aspectos que tienen que ver con la terminología y la sintaxis pues son los dos errores más comunes dentro de las secciones de la prueba; c. el desempeño en las pruebas de traducción e interpretación oficial es mayor en las convocatorias de los segundos semestres cuando se observa como promedio general; d. es importante reconocer de una mejor manera las herramientas que ofrece Internet para informarse y reconocer aspectos o rasgos terminológicos propios de las áreas del saber que hacen parte del examen. 
Desde el punto de vista conceptual, teórico y metodológico, la realización del presente estudio ha permitido reconocer de mejor manera las falencias, incongruencias y la acuciante necesidad de afinar la estrategia de evaluación en traducción oficial implementada en la Universidad de Antioquia, esto con miras a contribuir con la objetividad en el proceso evaluativo y definir de una mejor manera las necesidades y compromisos futuros que el medio académico tiene con la extensión en traducción. En cuanto al instrumento, puede decirse que la implementación de una estrategia de evaluación que se acomode a los parámetros y estándares profesionales y académicos es el primer paso hacia la objetividad en la revisión de traducciones. En este sentido, la plantilla de evaluación aún no está exenta de modificaciones, y es una herramienta fundamental e indispensable en el proceso de acreditación de los aspirantes al ETIO.

Desde un punto de vista académico y de formación profesional, se observa una necesidad de preparar o formar a los futuros profesionales en lenguas en técnicas de interpretación que les permitan salir a un mercado en el que la oferta para este tipo de oficio es baja a pesar de su buena remuneración.

Desde un punto de vista político, se observa que hace falta definir de una mejor manera los criterios profesionales de acceso al examen. El hecho de que la legislación colombiana aún no se haya dado a la tarea de legislar acerca de la profesión y de los exámenes de calificación de los aspirantes a este título, podría llegar a ser problemático en términos gremiales puesto que, luego de la revisión en la legislación acerca de traducción e interpretación oficial, aun cuando hay unas apelaciones constantes a los profesionales en esta área, no hay normas o reglas claras de juego en lo que respecta al ingreso a la misma o a la garantía de su calidad, etc. Tendría que pensarse en una modificación de la legislación en la que se incluyan algunas de las condiciones que posibiliten un mejor desempeño del traductor en el Examen, como su previa preparación en un campo que esté en relación con el área o una edad específica en la que tendría que presentarse al mismo, etc. 
Desde el punto de vista de los errores, la terminología se presenta como uno de los principales inconvenientes para los aspirantes al examen. Esta constatación, no siendo algo nuevo, permite pensar que es necesario que se refuerce mucho más el estudio y desarrollo de esta área en el país. La investigación, la formación y el desarrollo profesional en este aspecto son necesidades para nuestro contexto, lo que podría redundar en un mejor desempeño, no solo en este examen específico sino también a nivel laboral dentro de la profesión. Sin embargo, es importante comentar que, en vista de las condiciones del examen (acceso libre a internet, diccionarios, bases de datos, etc.) la dificultad en lo que concierne al tipo de error más común (terminológico) no está necesariamente relacionado con falta de conocimiento del candidato, sino que también podría ser de una pobre preparación en competencias estratégicas como por ejemplo en lo que tiene que ver con la búsqueda de información en la red... ¿Qué hace falta entonces en los procesos de formación o en los candidatos al examen? ¿Una mejor preparación en lo que tiene que ver con las estrategias de información y recolección de información a la hora de llevar a cabo una traducción?

Finalmente, es necesario, entre las comisiones al ETIO de Colombia, emplear estrategias profesionales para la revisión de las traducciones. No se trata simplemente de aplicar ciertos parámetros generales, sino estar encaminados a realizar todo un conglomerado de acciones que redunden en la adecuada revisión de la calidad de las traducciones de los profesionales en el país. 


\section{Referencias}

BACHMAN, Lyle. Fundamental Considerations in Language Testing. Oxford: Oxford: University Press, 1990.

CHAMOSA, José Luis. "Crítica y evaluación de traducciones: elementos para su discusión”. In: NISTAL, Fernández y GOZALO, José María Bravo. Aproximaciones a los estudios de traducción. (Eds.) Purificación. Valladolid: Universidad de Valladolid, 1997, p. 29-50.

CACHIN, Marie. La traduction. Paris: Électre - Éditions du Cercle de la Librairie, 2007.

Decreto 2257. Por el cual se reglamenta el Decretoley 382 del 19 de febrero de 1951, sobre intérpretes oficiales. Diario Oficial de Colombia. Consultado en: [www.leyex.com].

Decreto 382. Cargo de intérpretes oficiales. Diario Oficial de Colombia. Consultado el 29 de marzo de 2012 en: [http://www.cntv.org.co.]

Decreto 722. Por el cual se dictan normas sobre el ejercicio de la profesión de intérpretes oficiales. Consultado el 29 de marzo de 2012 en: [www.leyex.com].

DELISLE, Jean. La traduction raisonnée. Manuel d'initiation à la traduction professionnelle de l'anglais vers le français. Ottawa: Les Presses de l'Université d'Ottawa, 1992.

GOUADEC, Daniel. Paramètres de l'évaluation des traductions. Meta, 26 (2), 99-116, 1981.

GOUADEC, Daniel. Le traducteur, la traduction et l'entreprise. Paris: Afnor, 1989.

HOUSE, Juliane. Translation Quality Assessment: A Model Revisited. Tübingen: Gunter Narr Verlag, 1997. 
HURTADO, Amparo. "La didáctica de la traducción. Evolución y estado actual". In: NISTAL, Fernández y GOZALO, José María Bravo. Perspectivas de la traducción. Eds. Purificación. Valladolid: Universidad de Valladolid, 1995, p. 49-74.

HURTADO, Amparo. Traducción y traductología. Madrid: Cátedra, 2001.

KUSSMAUL, Paul. Training the Translator. Amsterdam: John Benjamins, 1995.

LAROSE, Robert. Méthodologie de l'évaluation des traductions. Meta, 43 (2) 163-86, 1998.

Ley 906 de 2004. Por la cual se expide el Código de Procedimiento Penal. Diario Oficial de Colombia. Consultado el 29 de marzo de 2012 en: [www.leyex.com].

Ley 962 de 2005. Disposiciones sobre racionalización de trámites y procedimientos administrativos de los organismos y entidades del Estado y de los particulares que ejercen funciones públicas o prestan servicios públicos. Diario Oficial de Colombia. Consultado el 29 de marzo de 2012 en: [www.leyex.com].

MARTÍNEZ, Nicole y HURTADO, Amparo. "Assessment in Translation Studies: Research Needs”. Meta, 46 (2), 272-287, 2001.

NEWMARK, Peter. A Textbook of Translation. Londres: Prentice Hall International, 1987.

NORD, Christiane. "El error en la traducción; categorías y evaluación". In: HURTADO, Amparo Albir. La enseñanza de la traducción. Ed. Castellón: Universidad Jaume I, 1996, 91-107.

ORDOÑEZ, Pilar. La evaluación de traductores e intérpretes jurados. Revisión teórica y análisis del tratamiento informativo de los candidatos a pruebas de acreditación y selección. Íkala 14: 57-82, 2009.

PARRA, Silvia. La revisión de traducciones en la traductología: aproximación a la práctica en el ámbito profesional mediante el estudio de casos y propuestas de investigación. Tesis doctoral, Universidad de Granada, 2005. 
PYM, Anthony. "Translation Error Analysis and the Interface with Language Teaching. Teaching Translation and Interpreting. Training, Talent and Experience". Papers from the First Language International Conference, Elsinore, Denmark, 31 May-2 June, 1991. (Eds.) C. Dollerup y A. Loddegaard. Amsterdam: John Benjamins, 1992, 279-288.

QUIROZ, Gabriel y ZULUAGA, Juan Felipe. Candidatos a traductor e intérprete oficial en Colombia profesionales u oportunistas. Babel, 60 (4), 514-538, 2014.

QUIROZ, Gabriel et al. Panorama general del traductor e intérprete oficial en Colombia. Núcleo, 25 (30), 2015.

QUIROZ, Gabriel et al. Estatus actual del traductor en Colombia. Editorial Universidad de Antioquia: Medellín-Colombia, 2015.

REISS, Katharina. Translation Criticism - The Potential and Limitations. Traducido por: Errol F, Rhodes. Manchester: St. Jerome Publishing, 2000.

SAE J2450. Translation Quality Metrics. Consultado en: [http://www.sae.org], 2001.

SAGER, Juan. "Quality and Standards - The Evaluation of Translations".In: SAGER, Juan. The Translator's Handbook. 1989, p. 91-102

STEJSKAL, Jiri. "International Certification Study: South America Revisited". The ATA Chronicle. 8 de marzo de 2013, 8-14.

UNE 15038. Servicios de traducción. Requisitos para la prestación del servicio. España: Madrid AENOR, 2006.

VÁZQUEZ-AYORA, Gerardo. Introducción a la traductología. Washington D.C.: Georgetown University Press, 1977.

WADDINGTON, Cristopher. Different Methods of Evaluating Student Translations: The Question of Validity. Meta 46 (2), 311-325, 2001. 
WILLIAMS, Malcolm. Translation Quality Assessment: An ArgumentationCentered Approach. Ottawa: University of Ottawa Press, 2005.

WILLIAMS, Malcolm. Translation Quality Assessment. Mutatis Mutandis, 2 (1). 3 - 23, 2009.

Recebido em: 04 de novembro de 2017 Aceito em: 03 de fevereiro de 2018 Publicado em: maio de 2018 